\title{
Nanoscale Surface Photovoltage Mapping of 2D Materials and Heterostructures by Illuminated Kelvin Probe Force Microscopy
}

Melinda J. Shearer, Ming-Yang Li, Lain-Jong Li, Song Jin, and Robert J Hamers

J. Phys. Chem. C, Just Accepted Manuscript • DOI: 10.1021/acs.jpcc.7b12579 • Publication Date (Web): 31 Jan 2018

Downloaded from http://pubs.acs.org on February 6, 2018

\section{Just Accepted}

"Just Accepted" manuscripts have been peer-reviewed and accepted for publication. They are posted online prior to technical editing, formatting for publication and author proofing. The American Chemical Society provides "Just Accepted" as a service to the research community to expedite the dissemination of scientific material as soon as possible after acceptance. "Just Accepted" manuscripts appear in full in PDF format accompanied by an HTML abstract. "Just Accepted" manuscripts have been fully peer reviewed, but should not be considered the official version of record. They are citable by the Digital Object Identifier (DOI®). "Just Accepted" is an optional service offered to authors. Therefore, the "Just Accepted" Web site may not include all articles that will be published in the journal. After a manuscript is technically edited and formatted, it will be removed from the "Just Accepted" Web site and published as an ASAP article. Note that technical editing may introduce minor changes to the manuscript text and/or graphics which could affect content, and all legal disclaimers and ethical guidelines that apply to the journal pertain. ACS cannot be held responsible for errors or consequences arising from the use of information contained in these "Just Accepted" manuscripts. 


\title{
Nanoscale Surface Photovoltage Mapping of 2D Materials and
}

\section{Heterostructures by Illuminated Kelvin Probe Force Microscopy}

\author{
Melinda J. Shearer, ${ }^{1}$ Ming-Yang Li, ${ }^{2,3}$ Lain-Jong $\mathrm{Li}^{2}{ }^{2}$ Song $\mathrm{Jin}^{1 *}$ and Robert J. Hamers ${ }^{1 *}$ \\ ${ }^{1}$ Department of Chemistry, University of Wisconsin-Madison, Madison, Wisconsin 53706, \\ United States \\ ${ }^{2}$ Physical Sciences and Engineering Division, King Abdullah University of Science and \\ Technology, Thuwal 23955-6900, Kingdom of Saudi Arabia \\ ${ }^{3}$ Research Center for Applied Sciences, Academia Sinica, Taipei 11529, Taiwan
}

\begin{abstract}
Nanomaterials are interesting for a variety of applications, such as optoelectronics and photovoltaics. However, they often have spatial heterogeneity, i.e. composition change or physical change in the topography or structure, which can lead to varying properties that would influence their applications. New techniques must be developed to understand and correlate spatial heterogeneity with changes in electronic properties. Here we highlight the technique of surface photovoltage-Kelvin probe force microscopy (SPV-KFM), which is a modified version of noncontact atomic force microscopy capable of imaging not only the topography and surface potential, but also the surface photovoltage on the nanoscale. We demonstrate its utility in probing monolayer $\mathrm{WSe}_{2}-\mathrm{MoS}_{2}$ lateral heterostructures, which form an ultrathin $\mathrm{p}$-n junction promising for photovoltaic and optoelectronic applications. We show surface photovoltage maps highlighting the different photoresponse of the two material regions as a result of the effective charge separation across this junction. Additionally, we study the variations between different
\end{abstract}


heterostructure flakes and emphasize the importance of controlling the synthesis and transfer of these materials to obtain consistent properties and measurements.

\section{INTRODUCTION}

Nanomaterials are interesting for solar energy conversion ${ }^{1-3}$ and other optoelectronic applications ${ }^{4-}$

${ }^{8}$ for a variety of reasons: tunability of electronic properties based on size, ${ }^{9-11}$ high surface area that allows for manipulation of properties via surface chemistry, ${ }^{12-14}$ and the ability to form unique heterojunctions due to minimization of lattice strain. ${ }^{15}$ However, many nanomaterials have spatial heterogeneity at the nanoscale that can lead to drastic variations in material properties. Spatial heterogeneity can involve both macroscopic properties, such as varying chemical composition and lack of uniformity in the size and shape as well as localized features, such as step edges, adsorbed atoms, and defects. The formation of heterostructures or doping of nanomaterials can also create spatially-varying properties based on changes in the chemical composition. All of these types of spatial heterogeneity can contribute to varying charge transfer properties, and it is critical to study how these changes influence the overall photoresponse of individual nanomaterials. Indeed, as new nanomaterials and heterostructures are synthesized, understanding charge transfer and dynamics within these materials is critically important, ${ }^{16}$ and high-resolution, high-throughput techniques with this capability are required.

Surface photovoltage (SPV) spectroscopy is a powerful technique for understanding the influence of surface electronic states on the photoresponse of materials, ${ }^{17-18}$ and has been used extensively to understand charge dynamics in a variety of semiconductor materials. ${ }^{19-22}$ This technique involves illuminating a semiconductor material and measuring the resulting surface 
photovoltage, typically using a metal-insulator-semiconductor configuration. A limitation of this technique, however, is its inability to measure spatial variation, which makes it impractical for studying individual nanostructures. One method in particular can help fill this void - surface photovoltage-Kelvin probe force microscopy (SPV-KFM), also referred to as illuminated KFM or microscopic SPV. With this method, standard Kelvin probe force microscopy is used to measure the surface potential of the material both in the dark and under illumination, the net difference of which creates surface photovoltage maps. It has so far been demonstrated on a small number of materials such as silicon wafers, ${ }^{23-24}$ organic solar cells ${ }^{25-28}$ and inorganic thin films, ${ }^{29-30}$ but viable demonstrations on individual nanostructures have been limited. ${ }^{31-33}$ Additionally, in a typical SPV-KFM setup, the sample is illuminated from below, thus requiring the use of a transparent conductive substrate, which is not always feasible or practical for device applications.

In this work, we have developed the SPV-KFM technique with illumination of the sample from above, and have demonstrated its efficacy on a monolayer lateral heterostructure of $\mathrm{WSe}_{2}$ $\mathrm{MoS}_{2} . \mathrm{WSe}_{2}$ and $\mathrm{MoS}_{2}$ are layered transition metal dichalcogenides $\left(\mathrm{MX}_{2}\right)$, which have received much research interest recently due to the ability to tune their properties based on the number ${ }^{34-35}$ and rotation ${ }^{36-37}$ of layers as well as their strong absorption at only a monolayer thickness. ${ }^{38}$ Therefore, they have been demonstrated for a variety of electronic, ${ }^{39-40}$ optoelectronic, ${ }^{41-42}$ solar energy conversion ${ }^{2}$ and catalysis applications. ${ }^{43-45}$ Additionally, these $2 \mathrm{D}$ materials can be synthesized as lateral heterostructures, allowing for the formation of covalently-bonded nanoscale p-n junctions that are promising for ultra-thin electronics. ${ }^{46-50}$ Extensive structural and electronic characterization of individual or heterojunction devices has been collected, ${ }^{46-48}$ but only rarely has there been information collected that combines charge transfer properties of these materials with spatial resolution. ${ }^{51-54}$ In order to see the effects of both heterostructure formation as well as 
variation among nanostructures, we have mapped the surface photovoltage of lateral $\mathrm{WSe}_{2}-\mathrm{MoS}_{2}$ heterostructures and revealed the heterogeneity in photoresponse between the two materials as a result of the charge transfer across the $p$ - $n$ junction that they form. We have also observed variations in the photoresponse of the $\mathrm{WSe}_{2}$ nanoflakes that can be attributed to changes in the substrate interaction due to the material transfer process. This work demonstrates the ability of the SPV-KFM technique to help determine the changes in optoelectronic properties due to heterojunctions and spatial heterogeneity on the nanoscale, and demonstrates its capability to observe changes within a lateral monolayer heterostructure for the first time.

\section{MATERIALS AND METHODS}

Synthesis of the $\mathrm{WSe}_{2}-\mathrm{MoS}_{2}$ Heterostructures.

The heterostructures were synthesized using a two-step CVD process reported previously. ${ }^{46}$ Briefly, during the first step for the growth of $\mathrm{WSe}_{2}, 0.6 \mathrm{~g}$ of $\mathrm{WO}_{3}$ powder in a quartz boat was placed in the center of the furnace, and Se powder was placed in a boat at the upstream side of the furnace and held at a temperature of $260{ }^{\circ} \mathrm{C}$ during the reaction. The sapphire substrate was placed at the downstream side of the CVD tube, just past the $\mathrm{WO}_{3}$ boat. The $\mathrm{WO}_{3}$ was heated to $925{ }^{\circ} \mathrm{C}$ at a pressure of 20 Torr for $15 \mathrm{~min}$ before letting the reaction cool down naturally to room temperature. During the reaction, $\mathrm{Ar}$ and $\mathrm{H}_{2}$ gas were flowed at 90 standard cubic centimeters per minute $(\mathrm{sccm})$ and $6 \mathrm{sccm}$, respectively. The sample was then placed into a separate furnace for the $\mathrm{MoS}_{2}$ growth. The setup of the boats for the $\mathrm{MoS}_{2}$ growth was the same as for the $\mathrm{WSe}_{2}$ synthesis, except now using $\mathrm{MoO}_{3}$ and $\mathrm{S}$ powders in place of $\mathrm{WO}_{3}$ and Se. The distance between the $\mathrm{MoO}_{3}$ boat and the $\mathrm{WSe}_{2}$ sample was about $9 \mathrm{~cm}$. The hot zone of the furnace was set to 755 ${ }^{\circ} \mathrm{C}$, while the $\mathrm{S}$ powder was held at $190{ }^{\circ} \mathrm{C}$ for $10 \mathrm{~min}$ and then naturally cooled to room 
temperature. Only Ar was flowed at a flow rate of $70 \mathrm{sccm}$ during this reaction, and the pressure was set to 40 Torr.

Transfer of $\mathrm{WSe}_{2}-\mathrm{MoS}_{2}$ Heterostructures.

The $\mathrm{WSe}_{2}-\mathrm{MoS}_{2}$ heterostructures were transferred from the sapphire growth substrate to native oxide coated degenerately-doped Si wafers via a standard poly(methylmethacrylate) (PMMA) transfer process (950 PMMA A4 MicroChem). PMMA was first spin-coated onto the surface of the sample, after which the entire sample was placed in $6 \mathrm{M} \mathrm{HF}$ solution to etch away the sapphire substrate. The remaining heterostructures covered in PMMA were removed from the HF, rinsed in DI water, and then transferred onto the Si substrate. The PMMA layer was then removed by placing the substrate into hot $\left(60^{\circ} \mathrm{C}\right)$ acetone for $30 \mathrm{~min}$. After the removal, the heterostructures were annealed at $300^{\circ} \mathrm{C}$ for approximately $2.5 \mathrm{~h}$ under vacuum $\left(10^{-3} \mathrm{Torr}\right)$.

Synthesis of the Individual $\mathrm{WSe}_{2}$ and $\mathrm{MoS}_{2}$ Monolayers.

The $\mathrm{WSe}_{2}$ and $\mathrm{MoS}_{2}$ monolayers were grown similarly to how they were grown for the heterostructure sample (see Synthesis of $\mathrm{WSe}_{2}-\mathrm{MoS}_{2}$ Heterostructures above).

Characterization of the $\mathrm{WSe}_{2}-\mathrm{MoS}_{2}$ Heterostructures.

The heterostructures were characterized using an optical microscope (Olympus BX51M), scanning electron microscope (LEO SUPRA 55 VP field-emission SEM) operated at $3 \mathrm{kV}$, and a Confocal Raman/Photoluminescence microscope/spectrometer (LabRAM Aramis). The Raman spectra and PL mapping measurements were collected using a $532 \mathrm{~nm}$ laser and a 100x microscope objective. 
Surface Photovoltage-Kelvin Probe Force Microscopy (SPV-KFM) Measurements.

The SPV-KFM measurements were carried out on an Agilent 5500 Atomic Force Microscope. The topography and the Kelvin probe measurements were collected simultaneously in single pass non-contact amplitude modulation mode. The Kelvin probe measurements were conducted using MikroMasch Pt coated Si tips (HQ:NSC18/Pt), which typically have a resonance frequency of 75 $\mathrm{kHz}$, force constant of $2.8 \mathrm{~N} / \mathrm{m}$, and a tip radius of $<30 \mathrm{~nm}$. In our setup, the DC offset bias was applied to the tip, $\mathrm{V}_{\mathrm{DC}}=\mathrm{V}_{\text {tip }}$. Therefore, $\mathrm{V}_{\text {tip }}=-\mathrm{CPD}$ and if $\mathrm{CPD}=\Phi_{\text {tip }}-\Phi_{\text {sample }}$, then $\mathrm{V}_{\text {tip }}=\Phi_{\text {sample }}$

$-\Phi_{\text {tip. }}{ }^{26}$ In this configuration, changes to the surface potential are correlated directly to the sign of the charge; i.e., a positive change in the surface potential corresponds to positive charging of the surface. The surface photovoltage aspect of the technique was implemented by directing a laser diode down onto the surface of the sample using a mirror. The laser diode for the $\mathrm{WSe}_{2}-\mathrm{MoS}_{2}$ experiments was from ThorLabs, Inc (LP405-SF10) and emits at $401.5 \mathrm{~nm}$ operated at $\sim 4 \mathrm{~mW}$. A collimator (ThorLabs, Inc. F280FC-A) was attached to the laser diode to get a large beam size of $3.3 \mathrm{~mm}$, which was then focused using a Newport lens with a focal length of $113 \mathrm{~mm}$ (KPX596C). The silicon test experiments were conducted using a laser diode that emits at $637.3 \mathrm{~nm}$ (Thorlabs, Inc model LP635-SF8) with another collimator (Thorlabs Model F280FC-B).

\section{RESULTS AND DISCUSSION}

Design and implementation of the SPV-KFM technique. Figure 1 illustrates a simplified schematic of the SPV-KFM setup. KFM involves the use of two lock-in amplifiers coupled with feedback controllers that can simultaneously measure both the topography and the surface potential of the sample relative to the tip by tracking the reflection of a laser off the cantilever onto a photodiode. Because the tip and the sample will, in general, have different work functions, when 
they are placed in electrical contact and brought close to each other a build-up of charge will occur as the Fermi levels equilibrate, known as the contact potential difference (CPD) ${ }^{55}$ In typical KFM, a DC voltage is applied to offset this CPD, which is then mapped as the surface potential (SP) of the material relative to the tip. ${ }^{55}$ The surface photovoltage (SPV) is defined as the change in local potential produced in response to optical excitation, which is measured experimentally by obtaining the CPD at each location in an image both in the absence of and in the presence of optical excitation from an external optical source; the difference between these images is a spatial map of the surface photovoltage.

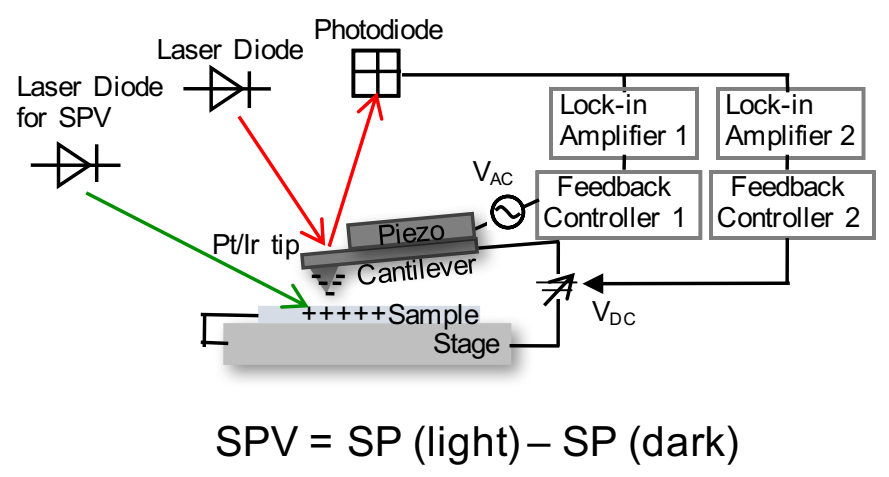

Figure 1. Schematic of the SPV-KFM setup showing the implementation of an additional laser diode to illuminate the sample from above. The general formula for calculating the surface photovoltage (SPV) is shown as well.

To measure SPV-KFM images, we modified a commercial AFM (Agilent 5500) by adding another laser diode (Thor Labs, Inc. LP405-SF 10, $401.5 \mathrm{~nm}, 4 \mathrm{~mW}$ ) with a collimator and a focusing lens to illuminate the sample from above (e.g., from the same side as the AFM tip). A custom-built part from Agilent containing a pre-drilled hole with a mirror to reflect the laser light down onto the surface of the sample was used, as shown in Figure S1 in the Supporting Information (SI). The laser intensity could be modulated digitally with computer control, thereby allowing for measurements of the surface potential in the light as well as in the dark. While in principle the 
optical illumination could be turned on and off at each point in an image, we found that better results were obtained by taking one AFM image with the laser on, and then re-scanning the same area with the laser off. From these two distinct images, the SPV maps can be calculated as the difference between these images.

To ensure that the SPV-KFM technique is working properly, we first measured the surface potential on $\mathrm{n}$-type and $\mathrm{p}$-type $\mathrm{Si}$ wafers of varying resistivities. Figure 2 shows the topography (Figure 2a) and the surface potential under illumination [SP (light)] (Figure 2b) of a native oxide coated n-type Si wafer of moderate resistivity $(\boldsymbol{\rho}=10 \Omega \bullet \mathrm{cm})$. The surface potential measured in the dark [SP (dark)] on this sample is shown in the SI Figure S2. Figure 2c shows two line profiles of surface potential measured in the light (blue line) and dark (green line). The net SPV for this material is positive. In general, n-type semiconductors exhibit upward band bending at their surfaces due to the presence of mid-gap surface states. ${ }^{56}$ When illuminated, the sub-surface electric field drives holes to the surface and shuttles electrons towards the bulk, such that the surface undergoes a shift toward more positive potentials. Thus, the positive SPV observed for n-type silicon is expected and has been reported previously. ${ }^{57}$ We also performed this measurement on p-type Si wafers, which exhibit downward band bending. As expected, a negative SPV response was observed, shown in Figure S3 in the SI. Figure S3 also shows plots of the average SPV measured for Si wafers with a variety of resistivities. These plots show that at very low or very high resistivities there is little SPV response from the $\mathrm{Si}$, and the maximum SPV is observed for $\mathrm{Si}$ with intermediate resistivities, which means that the $\mathrm{Si}$ is not too heavily doped (so that it is acting like a metal) or too lightly doped (so that it acts like an insulator). These results show that the surface potential and photopotentials that we measure experimentally with this SPV-KFM technique are in accordance with those expected when samples of known electronic structure are 
tested. After this validation, we can apply this technique to understanding materials of interest.

n-type Si, $\rho=10 \Omega^{*} \mathrm{~cm}$

a) Topography

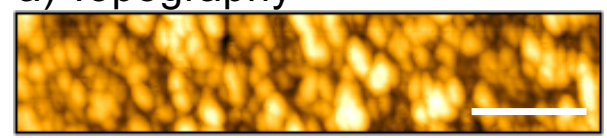

b) Surface Potential (SP) (light)

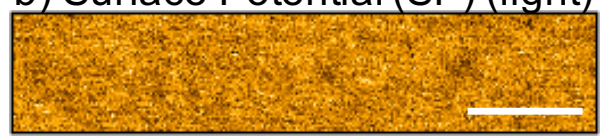

c) Line Profiles

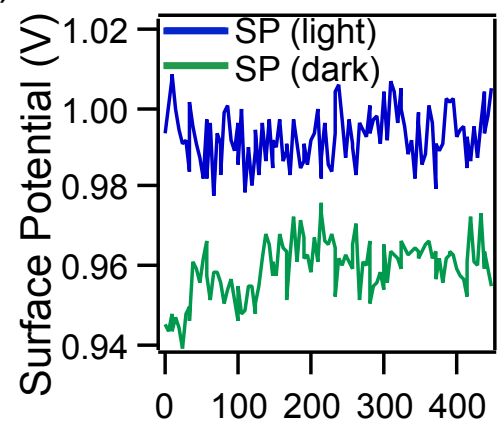

Distance $(\mathrm{nm})$

Figure 2. SPV-KFM data of a control n-type Si wafer coated with native oxide with a resistivity

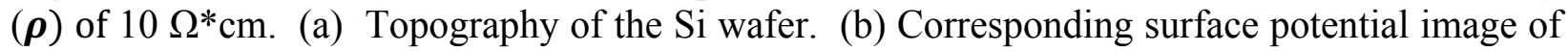
the Si under illumination with a $637.3 \mathrm{~nm}$ laser diode. (c) Line profiles of the surface potential with illumination [SP (light), blue line] and without illumination [SP (dark), green line]. There is a clear increase in surface potential under illumination vs. in the dark. Scale bars: $200 \mathrm{~nm}$.

Characterization of the $\mathrm{WSe}_{2}-\mathrm{MoS}_{2}$ Heterostructures. The monolayer lateral heterostructures of $\mathrm{WSe}_{2}-\mathrm{MoS}_{2}$ are typically grown on insulating substrates such as c-plane sapphire ${ }^{46}$ and must be transferred to other substrates for measurements requiring electrical contact. We synthesized $\mathrm{WSe}_{2}-\mathrm{MoS}_{2}$ heterostructures on sapphire substrates via chemical vapor deposition (CVD) and then transferred these heterostructures onto degenerately-doped n-type Si substrates (resistivity $=$ $0.007-0.02 \Omega \bullet \mathrm{cm}$ ) with native oxide coating using a method described previously ${ }^{46}$ (see Materials and Methods for procedures). Degenerately-doped n-type Si was chosen as the substrate because it will not exhibit any measureable photoresponse. Additionally, the technique requires the use of a substrate that is not insulating to help facilitate the Fermi level equilibration required to get high- 
resolution Kelvin probe surface potential images. Figure 3 shows the structural characterization of these $\mathrm{WSe}_{2}-\mathrm{MoS}_{2}$ heterostructures. Scanning electron microscopy (SEM) (Figure 3a) shows the heterostructures after transfer to the Si substrate. Many triangular features are clustered together and contain two distinct regions: a lighter inner triangle and a surrounding darker outer triangle. Figure $3 \mathrm{~b}$ and $3 \mathrm{c}$ show photoluminescence $(\mathrm{PL})$ spectra of the individual $\mathrm{WSe}_{2}$ and $\mathrm{MoS}_{2}$ regions, respectively. The peaks around $745 \mathrm{~nm}^{35,58}$ for $\mathrm{WSe}_{2}$ (Figure 3b) and $675 \mathrm{~nm}^{34}$ for $\mathrm{MoS}_{2}$ (Figure 3c) are at characteristic wavelengths for a monolayer of each material. Raman spectra (Figure S4 in SI) further confirm that both materials are one monolayer thick. For the $\mathrm{MoS}_{2}$ monolayer, the distance between the $A_{1 \mathrm{~g}}$ and the $\mathrm{E}_{2 \mathrm{~g}}$ peaks is approximately $18-19 \mathrm{~cm}^{-1}$, which is in agreement with reported literature values and much smaller than those for a bilayer or thicker films, which are usually between $21-25 \mathrm{~cm}^{-1} .{ }^{59-60}$ For the $\mathrm{WSe}_{2}$ monolayer, there is no peak at 308 $\mathrm{cm}^{-1}$, which only appears when $\mathrm{WSe}_{2}$ becomes a bilayer or thicker. ${ }^{61-62}$ To illustrate which regions of the heterostructure shown in Figure 3a correspond to each material $\left(\mathrm{WSe}_{2} \mathrm{vs}_{\mathrm{MoS}}\right)$, Figure $3 \mathrm{~d}$ and 3e show spatial maps of the photoluminescence intensity at emission wavelengths of 745 and $675 \mathrm{~nm}$, respectively. Figure $3 \mathrm{~d}$ shows that high fluorescence intensity is observed from triangular regions, while the spatial map at $675 \mathrm{~nm}$ in Figure 3e has a complementary appearance, with high fluorescence intensity in the regions between the triangles of Figure $3 \mathrm{~d}$. Based on the spatial distribution of fluorescence at each wavelength and the known emission spectra of $\mathrm{MoS}_{2}$ and $\mathrm{WSe}_{2}$, we conclude that the triangular regions are $\mathrm{WSe}_{2}$ monolayers and the regions surrounding the triangles are $\mathrm{MoS}_{2}$ monolayers. 


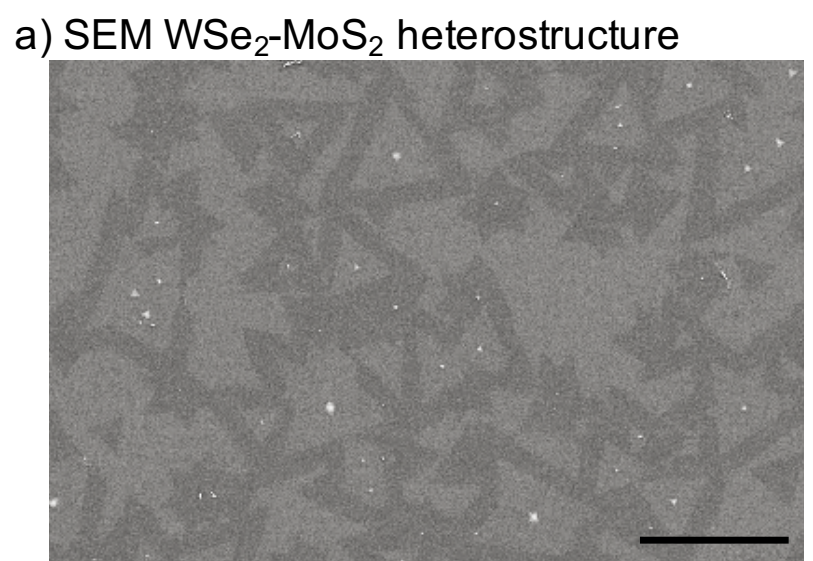

b) $\mathrm{WSe}_{2} \mathrm{PL}$

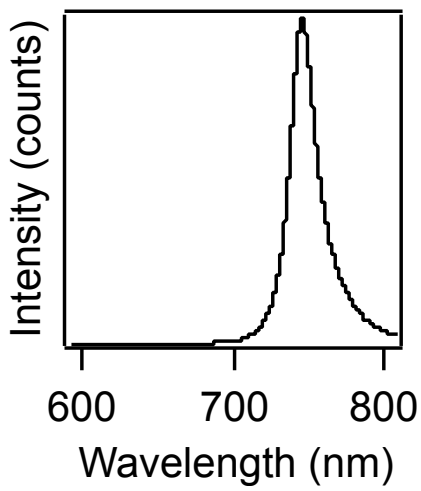

c) $\mathrm{MoS}_{2} \mathrm{PL}$

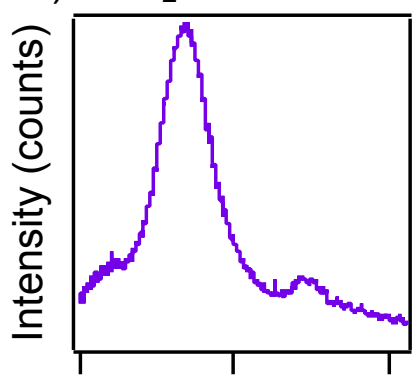

$\begin{array}{lll}600 & 700 \quad 800\end{array}$

Wavelength $(\mathrm{nm})$ e) PL map at $675 \mathrm{~nm}$
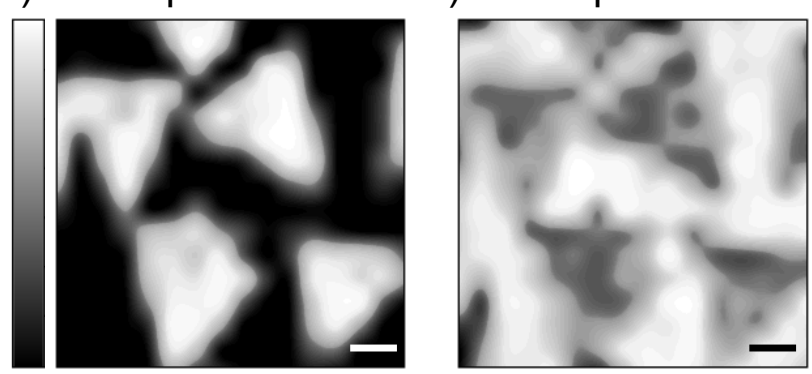

Figure 3. Structural characterization of the lateral $\mathrm{WSe}_{2}-\mathrm{MoS}_{2}$ heterostructures. (a) SEM image of the $\mathrm{WSe}_{2}-\mathrm{MoS}_{2}$ heterostructures on Si. Scale bar: $20 \mu \mathrm{m}$. (b) and (c) Photoluminescence (PL) spectra of the $\mathrm{WSe}_{2}$ region (black) at $745 \mathrm{~nm}$ and the $\mathrm{MoS}_{2}$ region (purple) at $675 \mathrm{~nm}$. (d) and (e) PL maps showing the emission corresponding to $\mathrm{WSe}_{2}$ at $745 \mathrm{~nm}$ (d) and $\mathrm{MoS}_{2}$ at $675 \mathrm{~nm}$ (e). These maps clearly show that the triangular flakes are $\mathrm{WSe}_{2}$ and the surrounding regions are $\mathrm{MoS}_{2}$. Scale bars: $2 \mu \mathrm{m}$.

SPV-KFM of the $\mathrm{WSe}_{2}-\mathrm{MoS}_{2}$ Heterostructures. Figure 4 displays the SPV-KFM data obtained from $\mathrm{WSe}_{2}-\mathrm{MoS}_{2}$ heterostructures that were grown on sapphire and then transferred onto n-type $\mathrm{Si}$ (resistivity $0.007-0.02 \Omega \bullet \mathrm{cm}$ ) as described above (see Materials and Methods for data collection details). Because of the high dopant concentration, such degenerately-doped Si substrate generates 
little to no SPV, as confirmed in Figure S3 in the SI and by further control measurements on a bare region of the Si substrate (Figure S5 in the SI). Figure 4a shows the topography image of the heterostructures, revealing few distinguishing features between the two materials in the heterostructure; several small folds and/or wrinkles that arise from the transfer from sapphire to the Si substrate are observed. Figure $4 \mathrm{~b}$ shows the surface potential map without illumination, revealing three brighter $\mathrm{WSe}_{2}$ triangular flakes surrounded by the darker $\mathrm{MoS}_{2}$. In this image, there is very strong contrast in the surface potential as a result of the chemical composition, which is not apparent in the topography image. The regions of $\mathrm{WSe}_{2}$ and $\mathrm{MoS}_{2}$ are labeled by correlating with the PL maps shown in Figure 3. Because of the already established connection between shape and composition, we can distinguish between the two materials in the KFM measurements.

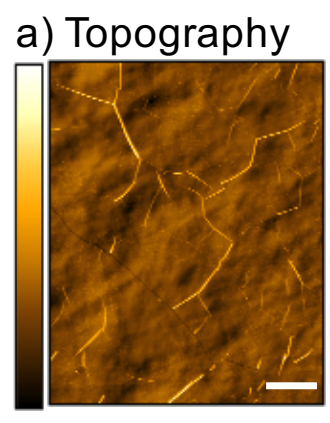

d) Net SPV

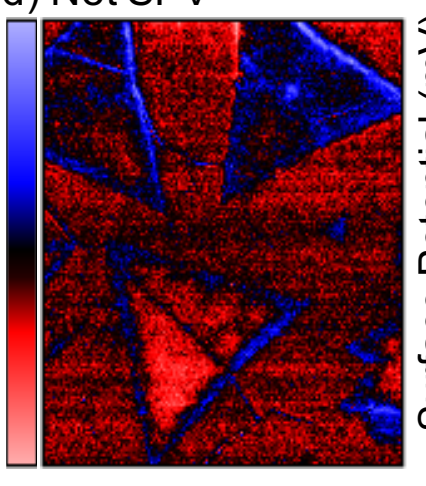

b) SP (dark)

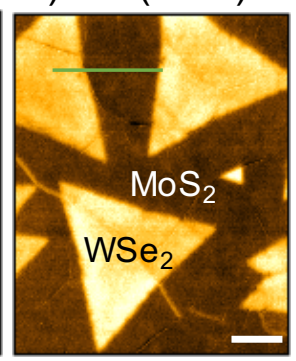

e) Line Profiles c) SP (light)
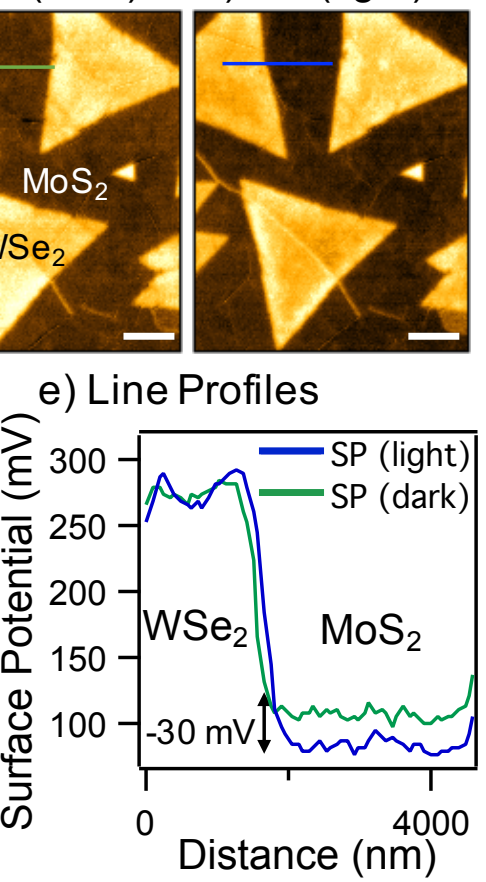

Figure 4. SPV-KFM maps of the $\mathrm{WSe}_{2}-\mathrm{MoS}_{2}$ heterostructures on n-type Si of resistivity $0.007-$ $0.02 \Omega * \mathrm{~cm}$. (a) Topography image of the $\mathrm{WSe}_{2}-\mathrm{MoS}_{2}$ heterostructures. (b) SP map taken with no illumination of the heterostructures. (c) SP map under illumination of the heterostructures. (d) Net SPV, calculated by subtracting the SP (dark) image from the SP (light) image. (e) Line 
profiles of SP (light) in blue and SP (dark) in green corresponding to the traces in (b) and (c) marked with green and blue lines. Scale bars: $2 \mu \mathrm{m}$.

Work function values of these materials were not calculated, as KFM performed in ambient conditions generally does not provide accurate work function values, due to many factors such as surface adsorbates, tip conditions, etc. However, based on the convention adopted herein (see Materials and Methods), we can use the equation $\mathrm{V}_{\text {tip }}=\Phi_{\text {sample }}-\Phi_{\text {tip }}$ to understand the work function differences between the two materials. The $\mathrm{WSe}_{2}$ flakes have a larger surface potential than the surrounding $\mathrm{MoS}_{2}$, based on the color scale shown in Figure 4b. This indicates that the work function of $\mathrm{WSe}_{2}$ is larger than that of $\mathrm{MoS}_{2}$. This work function difference would facilitate charge transfer between the materials, which will become more apparent under illumination.

Figure 4c shows the surface potential map under illumination with $401.5 \mathrm{~nm}$ light. In order to more easily see the differences in surface potential induced by illumination, Figure $4 \mathrm{~d}$ shows the net SPV image, obtained by subtracting the SP (dark) image from the SP (light) image. The SPV image of Figure $4 \mathrm{~d}$ has a color bar in which black has been set as zero SPV, with positive SPV appearing blue and negative SPV appearing red. To complement this image, Figure 4e shows the surface potential line profiles measured along the green line in Figure $4 \mathrm{~b}$ and the blue line in Figure 4c. It is clear from this data that there is an average surface photovoltage of approximately $-30 \mathrm{mV}$ for the $\mathrm{MoS}_{2}$ regions of the heterostructures, indicating an accumulation of negative charge when under illumination. This negative charge build-up could be a result of electron transfer across either the lateral p-n WSe $-\mathrm{MoS}_{2}$ junction or the $\mathrm{MoS}_{2}-\mathrm{Si}$ substrate interface. Different $\mathrm{WSe}_{2}$ regions themselves exhibit some variability in the SPV signal, but the average SPV signal was close to $0 \mathrm{mV}$, indicating no measureable photoresponse. This result is similar to that seen in $\mathrm{WSe}_{2}$ heterostructure field-effect transistor (FET) devices, where the $\mathrm{WSe}_{2}$ has been demonstrated to have lower mobility than $\mathrm{MoS}_{2}{ }^{63}$ and is the limiting step of charge transport. ${ }^{64}$ However, since 
the surface potentials of $\mathrm{WSe}_{2}$ and $\mathrm{MoS}_{2}$ are different, there must be an electric field at the interface of these two materials. This electric field provides a lateral driving force for separation of electrons and holes that is induced by the surface potential difference of the two materials at the junction. These results show that the SPV-KFM technique provides a way to directly probe the spatiallyvarying charge transfer properties when the system is driven away from equilibrium by illumination.

We also observed that different $\mathrm{WSe}_{2}$ flakes show variations in their SPV responses. For example, the bottom triangle shown in Figure $4 \mathrm{~d}$ has strong negative SPV, while the other two $\mathrm{WSe}_{2}$ triangles have nearly zero or slightly positive SPV. The lack of net photoresponse from these regions could be a result of offsetting processes, such as hole transfer across the $\mathrm{WSe}_{2}-\mathrm{MoS}_{2}$ junction in conjunction with electron transfer from the $\mathrm{Si}$ to the $\mathrm{WSe}_{2}$, or a result of carriers with limited mobility in $\mathrm{WSe}_{2}$ observed previously ${ }^{63}$ that will recombine before being efficiently separated. Because the samples were transferred from the growth substrate (sapphire) to another substrate ( $\mathrm{Si}$ ), the 2D layers observed are not epitaxially bound or definitively oriented with respect to the underlying substrate. The different photoresponse from nominally identical $\mathrm{WSe}_{2}$ triangles may arise from the variations of the interface between the structures and the underlying substrate. It is difficult to control the transfer to the Si substrate precisely, evident from the folds and wrinkles that are clearly present in the topography image in Figure 4a.

To further understand the SPV-KFM data, we have measured control samples of just WSe $\mathrm{S}_{2}$ and $\mathrm{MoS}_{2}$ monolayers on the same degenerately-doped Si substrate. As shown in Figures S6 and $\mathrm{S} 7$ in the SI, these measurements show a large positive SPV for the $\mathrm{WSe}_{2}$ monolayer, and a small negative SPV for the $\mathrm{MoS}_{2}$ monolayer. The overall signs of the SPV remain the same, but the photoresponse appears to be suppressed for the $\mathrm{WSe}_{2}$ and enhanced for the $\mathrm{MoS}_{2}$ when in a 
heterostructure as compared to the two materials when separate. These results further indicate that the photoresponse of the heterostructures is partially a result of the interaction between these two materials at the lateral junction.

We have further studied the SPV maps of a $\mathrm{WSe}_{2}-\mathrm{MoS}_{2}$ heterostructure that has been transferred to a p-type Si substrate that is not degenerately doped, as shown in Figure 5. A control scan taken on just the substrate, shown in Figure S8 in the SI, reveals the strong negative SPV exhibited by the substrate, typical for a p-type material of moderate resistivity (Figure S3 in the SI). Figure 5a shows the SP (dark) of one corner of a $\mathrm{WSe}_{2}$ flake with $\mathrm{MoS}_{2}$ surrounding it. The p-type Si substrate is also visible in this image beyond the boundary of the $\mathrm{MoS}_{2}$. Figure $5 \mathrm{~b}$ displays the SP (light) of the same area of the heterostructure, and the net SPV is shown in Figure $5 \mathrm{c}$, illustrating the stark difference in photoresponse between the two materials and the substrate. To highlight this further, surface potential line profiles have been plotted in Figure 5d showing the change in SP in the dark (green) and under illumination (blue) for the $\mathrm{WSe}_{2}, \mathrm{MoS}_{2}$, and p-Si regions traced along the green and blue lines in Figure 5a, b. From this line profile, it is apparent that the $\mathrm{SPV}$ in the $\mathrm{WSe}_{2}$ region is still approximately $0 \mathrm{mV}$, while the $\mathrm{SPV}$ in the $\mathrm{MoS}_{2}$ region is now $-110 \mathrm{mV}$. However, the $\mathrm{p}-\mathrm{Si}$ substrate is generating an SPV of $-70 \mathrm{mV}$. Therefore, we are likely measuring the SPV for both the $\mathrm{MoS}_{2}$ and the underlying Si substrate in the $\mathrm{MoS}_{2}$ region, resulting in the enhanced negative SPV signal compared to that shown in Figure 4e collected on the degenerately-doped n-type Si substrate. Interestingly, there is no corresponding change in the SPV for the $\mathrm{WSe}_{2}$, which suggests different interactions with the Si substrate are occurring between the two materials; perhaps the electrons moving to the surface of the p-type Si are combining with holes in the $\mathrm{WSe}_{2}$ generated when electrons move across the junction to the $\mathrm{MoS}_{2}$. Regardless, this comparison between the SPV data collected on different Si substrates makes it 
clear that the Si substrate itself does not dominate the photoresponse observed in Figure 4. The changes in the surface potential and the photoinduced changes in surface potential are driven by the differences in the intrinsic properties of these $\mathrm{MX}_{2}$ materials. The substrate has some effect on the absolute sign of the surface potential, but the difference between two distinct $\mathrm{MX}_{2}$ materials is largely independent of the nature of the substrate, particularly when the samples are transferred to the substrate.

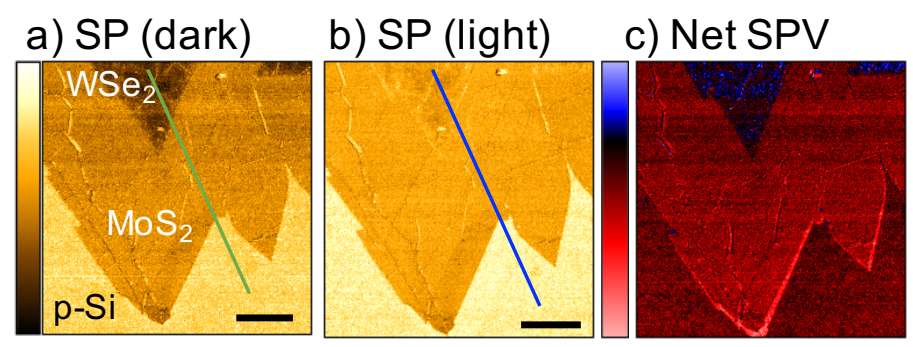

d) Line Profile

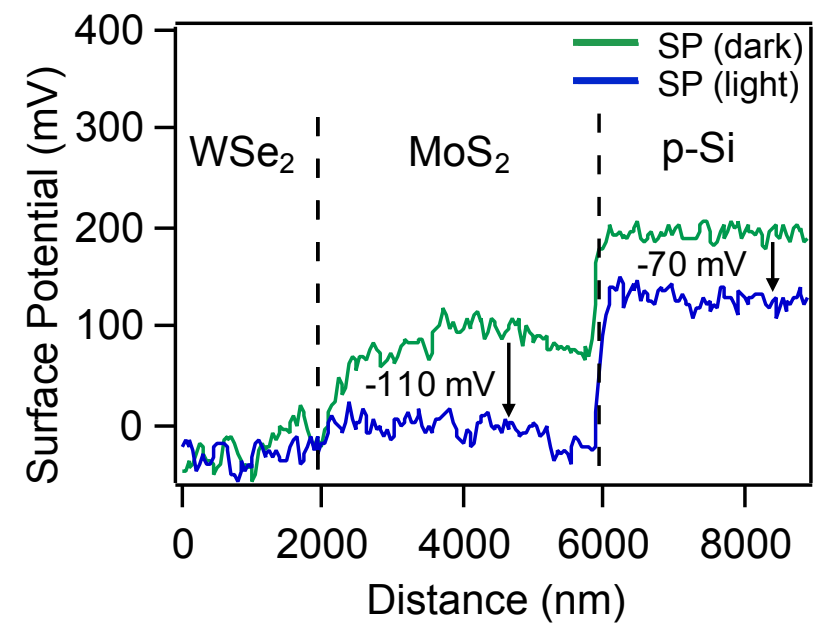

Figure 5. SPV-KFM maps of a $\mathrm{WSe}_{2}-\mathrm{MoS}_{2}$ heterostructure on p-type Si substrate with moderate resistivity. (a) $\mathrm{SP}$ of the $\mathrm{WSe}_{2}-\mathrm{MoS}_{2}$ heterostructure in the dark. The regions of $\mathrm{WSe}_{2}, \mathrm{MoS}_{2}$, and substrate (p-Si) are labeled. (b) SP under illumination of the same area of the heterostructure as shown in a. (c) Net SPV of the heterostructure. (d) Line profiles of SP (light) in blue and SP (dark) in green corresponding to the traces in (a) and (b) marked with green and blue lines. Scale bars: $2 \mu \mathrm{m}$.

As mentioned earlier, the SPV-KFM signal can be affected by the interface between the substrate and the $\mathrm{MX}_{2}$ material. For example, poor contact to the substrate could perhaps inhibit charge transfer between the two, which would influence the observed photoresponse. However, 
we believe that the variations observed between samples due to poor contact would be a reflection of the substrate interaction with the $\mathrm{MX}_{2}$ material, and could help to elucidate how the material would operate in an actual device, particularly when the sample must be transferred to a different substrate. To that end, there could be variation between samples that are a result of differences in the transfer process or interaction between substrate and material. However, three different samples that were prepared in a similar manner at different times all exhibited the same sign for the photoresponse for the individual $\mathrm{MX}_{2}$ materials, indicating that some local variations will exist between samples, but generally the result is reproducible among different samples on different days.

\section{CONCLUSION}

Our results demonstrate SPV-KFM as a useful technique for correlating spatial heterogeneity and photoresponse of individual nanostructures and in heterojunctions, specifically of 2D materials. We have validated the technique using Si wafers with known doping type and resistivity and studied a complex structure, the monolayer lateral heterostructure of $\mathrm{WSe}_{2}-\mathrm{MoS}_{2}$. SPV-KFM has revealed that the two materials have quite distinct photoresponses, likely as a result of the $\mathrm{p}-\mathrm{n}$ junction that can effectively separate charges between the two materials in the heterostructure. Interestingly, the photoresponse seems to be relatively uniform for $\mathrm{MoS}_{2}$ at $-30 \mathrm{mV}$ but varies for $\mathrm{WSe}_{2}$. Differences in the photoresponse between neighboring $\mathrm{WSe}_{2}$ flakes reveal the influence of varying substrate interaction as a result of transferring the as-grown samples to a conductive substrate. This SPV-KFM technique is useful for understanding charge transfer and accumulation in 2D materials and nanomaterials in general, and elucidating the effects of spatial heterogeneity 
and sample transfer on the photoresponse. Understanding these influences on properties will lead to better design of nanostructures for optoelectronic and photovoltaic applications.

\section{SUPPORTING INFORMATION}

The supporting information is available free of charge at the ACS Publications website. It contains additional SPV-KFM data for silicon controls, Raman spectra of the heterostructures, SPV-KFM, Raman, and PL data for a $\mathrm{WSe}_{2}$ monolayer and a $\mathrm{MoS}_{2}$ monolayer.

\section{AUTHOR INFORMATION \\ Corresponding Author \\ Correspondence should be addressed to R.J.H. (rjhamers@chem.wisc.edu) and S.J. (jin@chem.wisc.edu).}

\section{ACKNOWLEDGEMENT}

This work was supported by the Department of Energy, Office of Basic Energy Sciences, Division of Materials Science and Engineering, under Award DE-FG02-09ER46664. M.J.S. also thanks the National Science Foundation Graduate Research Fellowship Program under Grant No. DGE1256259 for support. Support was also provided by the Graduate School and the Office of the Vice Chancellor for Research and Graduate Education at the University of Wisconsin-Madison with funding from the Wisconsin Alumni Research Foundation.

\section{REFERENCES}

1. Kamat, P. V. Meeting the Clean Energy Demand: Nanostructure Architectures for Solar Energy Conversion. J. Phys. Chem. C 2007, 111, 2834-2860. 
2. $Y u, X$.; Sivula, K. Toward Large-Area Solar Energy Conversion with Semiconducting 2D Transition Metal Dichalcogenides. ACS Energy Lett. 2016, 1, 315-322.

3. Selinsky, R. S.; Ding, Q.; Faber, M. S.; Wright, J. C.; Jin, S. Quantum Dot Nanoscale Heterostructures for Solar Energy Conversion. Chem. Soc. Rev. 2013, 42, 2963-2985.

4. Lu, W.; Lieber, C. M. Nanoelectronics from the Bottom Up. Nat. Mater. 2007, 6, 841850.

5. Eaton, S. W.; Fu, A.; Wong, A. B.; Ning, C.-Z.; Yang, P. Semiconductor Nanowire Lasers. Nat. Rev. Mater. 2016, 1, 16028.

6. Fu, Y.; Zhu, H.; Stoumpos, C. C.; Ding, Q.; Wang, J.; Kanatzidis, M. G.; Zhu, X.; Jin, S. Broad Wavelength Tunable Robust Lasing from Single-Crystal Nanowires of Cesium Lead Halide Perovskites (CsPbX 3 , X = Cl, Br, I). ACS Nano 2016, 10, 7963-7972.

7. Chen, B.; Pradhan, N.; Zhong, H. From Large-Scale Synthesis to Lighting Device Applications of Ternary I-III-VI Semiconductor Nanocrystals: Inspiring Greener Material Emitters. J. Phys. Chem. Lett. 2018, 9, 435-445.

8. Zaiats, G.; Kinge, S.; Kamat, P. V. Origin of Dual Photoluminescence States in ZnS-CulnS 2 Alloy Nanostructures. J. Phys. Chem. C 2016, 120, 10641-10646.

9. Murray, C. B.; Kagan, C. R.; Bawendi, M. G. Synthesis and Characterization of Monodisperse Nanocrystals and Close-Packed Nanocrystal Assemblies. Annu. Rev. Mater. Sci. 2000, 30, 545-610.

10. Alivisatos, A. P. Semiconductor Clusters, Nanocrystals, and Quantum Dots. Science 1996, 271, 933-937.

11. Robel, I.; Kuno, M.; Kamat, P. V. Size-Dependent Electron Injection from Excited CdSe Quantum Dots into $\mathrm{TiO}_{2}$ Nanoparticles. J. Am. Chem. Soc. 2007, 129, 4136-4137.

12. Balakrishnan, S. K.; Kamat, P. V. Ligand Assisted Transformation of Cubic $\mathrm{CsPbBr}_{3}$ Nanocrystals into Two-Dimensional $\mathrm{CsPb}_{2} \mathrm{Br}_{5}$ Nanosheets. Chem. Mater. 2018, 30, 74-78.

13. Ding, Q.; Czech, K. J.; Zhao, Y.; Zhai, J.; Hamers, R. J.; Wright, J. C.; Jin, S. Basal-Plane Ligand Functionalization on Semiconducting $2 \mathrm{H}-\mathrm{MoS}_{2}$ Monolayers. ACS Appl. Mat. Interfaces 2017, 9, 12734-12742.

14. Hines, D. A.; Kamat, P. V. Recent Advances in Quantum Dot Surface Chemistry. ACS Appl. Mat. Interfaces 2014, 6, 3041-3057.

15. Bierman, M. J.; Jin, S. Potential Applications of Hierarchical Branching Nanowires in Solar Energy Conversion. Energy Environ. Sci. 2009, 2, 1050-1059.

16. Kamat, P. V. Manipulation of Charge Transfer across Semiconductor Interface. A Criterion That Cannot Be Ignored in Photocatalyst Design. J. Phys. Chem. Lett. 2012, 3, 663-672. 17. Kronik, L.; Shapira, Y. Surface Photovoltage Spectroscopy of Semiconductor Structures: At the Crossroads of Physics, Chemistry and Electrical Engineering. Surf. Interface Anal. 2001, 31, 954-965.

18. Clabes, J.; Henzler, M. Determination of Surface States on Si(111) by Surface Photovoltage Spectroscopy. Phys. Rev. B 1980, 21, 625-631.

19. Osterloh, F. E.; Holmes, M. A.; Zhao, J.; Chang, L.; Kawula, S.; Roehling, J. D.; Moulé, A. J. P3HT:PCBM Bulk-Heterojunctions: Observing Interfacial and Charge Transfer States with Surface Photovoltage Spectroscopy. J. Phys. Chem. C 2014, 118, 14723-14731. 
20. Dittrich, T.; Awino, C.; Prajongtat, P.; Rech, B.; Lux-Steiner, M. C. Temperature Dependence of the Band Gap of $\mathrm{CH}_{3} \mathrm{NH}_{3} \mathrm{Pbl}_{3}$ Stabilized with PMMA: A Modulated Surface Photovoltage Study. J. Phys. Chem. C 2015, 119, 23968-23972.

21. Kedem, N.; Kulbak, M.; Brenner, T. M.; Hodes, G.; Cahen, D. Type-Inversion as a Working Mechanism of High Voltage $\mathrm{MAPbBr}_{3}(\mathrm{Cl})$-Based Halide Perovskite Solar Cells. Phys. Chem. Chem. Phys. 2017, 19, 5753-5762.

22. Rodríguez-Pérez, M.; Canto, E.; García-Rodríguez, R.; De Denko, A. T.; Oskam, G.; Osterloh, F. E. Surface Photovoltage Spectroscopy Resolves Interfacial Charge Separation Efficiencies in ZnO Dye-Sensitized Solar Cells. J. Phys. Chem. C 2018, In press, DOI: 10.1021/acs.jpcc.7b11727.

23. Takihara, M.; Igarashi, T.; Ujihara, T.; Takahashi, T. Photovoltage Mapping on Polycrystalline Silicon Solar Cells by Kelvin Probe Force Microscopy with Piezoresistive Cantilever. Jpn. J. Appl. Phys., Part 1 2007, 46, 5548-5551.

24. Takahashi, T. Photoassisted Kelvin Probe Force Microscopy on Multicrystalline Si Solar Cell Materials. Jpn. J. Appl. Phys. 2011, 50, 08LA05.

25. Shao, G.; Glaz, M. S.; Ma, F.; Ju, H.; Ginger, D. S. Intensity-Modulated Scanning Kelvin Probe Microscopy for Probing Recombination in Organic Photovoltaics. ACS Nano 2014, 8, 10799-10807.

26. Fuchs, F.; Caffy, F.; Demadrille, R.; Mélin, T.; Grévin, B. High-Resolution Kelvin Probe Force Microscopy Imaging of Interface Dipoles and Photogenerated Charges in Organic DonorAcceptor Photovoltaic Blends. ACS Nano 2016, 10, 739-746.

27. Maturová, K.; Kemerink, M.; Wienk, M. M.; Charrier, D. S. H.; Janssen, R. A. J. Scanning Kelvin Probe Microscopy on Bulk Heterojunction Polymer Blends. Adv. Funct. Mater. 2009, 19, 1379-1386.

28. Palermo, V.; Palma, M.; Samorì, P. Electronic Characterization of Organic Thin Films by Kelvin Probe Force Microscopy. Adv. Mater. 2006, 18, 145-164.

29. Mesa, F.; Chamorro, W.; Vallejo, W.; Baier, R.; Dittrich, T.; Grimm, A.; Lux-Steiner, M. C.; Sadewasser, $\mathrm{S}$. Junction Formation of $\mathrm{Cu}_{3} \mathrm{BiS}_{3}$ Investigated by Kelvin Probe Force Microscopy and Surface Photovoltage Measurements. Beilstein J. Nanotechnol. 2012, 3, 277-284.

30. Polak, L.; Rector, J. H.; Slaman, M. J.; Wijngaarden, R. J. NaTaO 3 Photoanode for BiasFree Water Splitting: A Photo-Electrochemical and Kelvin Probe Surface Photovoltage Study. J. Phys. Chem. C 2016, 120, 23559-23565.

31. Batra, Y.; Deepa, R.; Bodh Raj, M. Scanning Kelvin Probe Investigations of $\mathrm{TiO}_{2}$ Nanoparticles/Poly(3-Hexylthiophene) Hybrid Blends. Appl. Phys. Express 2013, 6, 041602.

32. Wu, M.-C.; Liao, H.-C.; Cho, Y.-C.; Tóth, G.; Chen, Y.-F.; Su, W.-F.; Kordás, K. Photo-Kelvin Probe Force Microscopy for Photocatalytic Performance Characterization of Single Filament of $\mathrm{TiO}_{2}$ Nanofiber Photocatalysts. J. Mater. Chem. A 2013, 1, 5715-5720.

33. Gupta, S.; Batra, Y.; Mehta, B.; Satsangi, V. Study of Charge Separation and Interface Formation in a Single Nanorod $\mathrm{CdS}-\mathrm{Cu}_{x} \mathrm{~S}$ Heterojunction Solar Cell Using Kelvin Probe Force Microscopy. Nanotechnology 2013, 24, 255703.

34. Mak, K. F.; Lee, C.; Hone, J.; Shan, J.; Heinz, T. F. Atomically Thin MoS 2 : A New Direct-Gap Semiconductor. Phys. Rev. Lett. 2010, 105, 136805.

35. Zhao, W. J.; Ghorannevis, Z.; Chu, L. Q.; Toh, M. L.; Kloc, C.; Tan, P. H.; Eda, G. Evolution of Electronic Structure in Atomically Thin Sheets of WS ${ }_{2}$ and WSe ${ }_{2}$. ACS Nano 2013, 7, 791-797. 
36. Puretzky, A. A.; Liang, L.; Li, X.; Xiao, K.; Sumpter, B. G.; Meunier, V.; Geohegan, D. B. Twisted $\mathrm{MoSe}_{2}$ Bilayers with Variable Local Stacking and Interlayer Coupling Revealed by LowFrequency Raman Spectroscopy. ACS Nano 2016, 10, 2736-2744.

37. Shearer, M. J.; Samad, L.; Zhang, Y.; Zhao, Y.; Puretzky, A.; Eliceiri, K. W.; Wright, J. C.; Hamers, R. J.; Jin, S. Complex and Noncentrosymmetric Stacking of Layered Metal Dichalcogenide Materials Created by Screw Dislocations. J. Am. Chem. Soc. 2017, 139, 34963504.

38. Kozawa, D.; Kumar, R.; Carvalho, A.; Kumar Amara, K.; Zhao, W.; Wang, S.; Toh, M.; Ribeiro, R. M.; Castro Neto, A. H.; Matsuda, K., et al. Photocarrier Relaxation Pathway in TwoDimensional Semiconducting Transition Metal Dichalcogenides. Nat. Commun. 2014, 5, 4543. 39. Manzeli, S.; Ovchinnikov, D.; Pasquier, D.; Yazyev, O. V.; Kis, A. 2D Transition Metal Dichalcogenides. Nat. Rev. Mater. 2017, 2, 17033.

40. Schaibley, J. R.; Yu, H.; Clark, G.; Rivera, P.; Ross, J. S.; Seyler, K. L.; Yao, W.; Xu, X. Valleytronics in 2D Materials. Nat. Rev. Mater. 2016, 1, 16055.

41. Mak, K. F.; Shan, J. Photonics and Optoelectronics of 2D Semiconductor Transition Metal Dichalcogenides. Nat. Photon. 2016, 10, 216-226.

42. Samad, L.; Bladow, S. M.; Ding, Q.; Zhuo, J.; Jacobberger, R. M.; Arnold, M. S.; Jin, S. Layer-Controlled Chemical Vapor Deposition Growth of $\mathrm{MoS}_{2}$ Vertical Heterostructures via Van Der Waals Epitaxy. ACS Nano 2016, 10, 7039-7046.

43. Ding, Q.; Song, B.; Xu, P.; Jin, S. Efficient Electrocatalytic and Photoelectrochemical Hydrogen Generation Using $\mathrm{MoS}_{2}$ and Related Compounds. Chem 2016, 1, 699-726.

44. Putungan, D. B.; Lin, S.-H.; Kuo, J.-L. A First-Principles Examination of Conducting Monolayer $1 \mathrm{~T}^{\prime}-\mathrm{MX}_{2}(\mathrm{M}=\mathrm{Mo}, \mathrm{W} ; \mathrm{X}=\mathrm{S}, \mathrm{Se}, \mathrm{Te})$ : Promising Catalysts for Hydrogen Evolution Reaction and Its Enhancement by Strain. Phys. Chem. Chem. Phys. 2015, 17, 21702-21708. 45. Zhang, X. W.; Meng, F.; Mao, S.; Ding, Q.; Shearer, M. J.; Faber, M. S.; Chen, J. H.; Hamers, R. J.; Jin, S. Amorphous $\mathrm{MoS}_{x} \mathrm{Cl}_{y}$ Electrocatalyst Supported by Vertical Graphene for Efficient Electrochemical and Photoelectrochemical Hydrogen Generation. Energy Environ. Sci. 2015, 8, 862-868.

46. Li, M.-Y.; Shi, Y.; Cheng, C.-C.; Lu, L.-S.; Lin, Y.-C.; Tang, H.-L.; Tsai, M.-L.; Chu, C.-W.; Wei, K.-H.; He, J.-H., et al. Epitaxial Growth of a Monolayer WSe $-\mathrm{MoS}_{2}$ Lateral P-N Junction with an Atomically Sharp Interface. Science 2015, 349, 524-528.

47. Gong, Y.; Lin, J.; Wang, X.; Shi, G.; Lei, S.; Lin, Z.; Zou, X.; Ye, G.; Vajtai, R.; Yakobson, B. I., et al. Vertical and in-Plane Heterostructures from $\mathrm{WS}_{2} / \mathrm{MoS}_{2}$ Monolayers. Nat. Mater. 2014, 13, 1135-1142.

48. Duan, X.; Wang, C.; Shaw, J. C.; Cheng, R.; Chen, Y.; Li, H.; Wu, X.; Tang, Y.; Zhang, Q.; Pan, A., et al. Lateral Epitaxial Growth of Two-Dimensional Layered Semiconductor Heterojunctions. Nat. Nanotechnol. 2014, 9, 1024-1030.

49. Zhang, X.-Q.; Lin, C.-H.; Tseng, Y.-W.; Huang, K.-H.; Lee, Y.-H. Synthesis of Lateral Heterostructures of Semiconducting Atomic Layers. Nano Lett. 2015, 15, 410-415.

50. Feng, L.-p.; Su, J.; Liu, Z.-t. Characteristics of Lateral and Hybrid Heterostructures Based on Monolayer MoS 2 : A Computational Study. Phys. Chem. Chem. Phys. 2017, 19, 4741-4750. 51. Son, Y.; Li, M.-Y.; Cheng, C.-C.; Wei, K.-H.; Liu, P.; Wang, Q. H.; Li, L.-J.; Strano, M. S. Observation of Switchable Photoresponse of a Monolayer WSe $\mathrm{MoS}_{2}$ Lateral Heterostructure via Photocurrent Spectral Atomic Force Microscopic Imaging. Nano Lett. 2016, 16, 3571-3577. 
52. Li, Y.; Xu, C. Y.; Wang, J. Y.; Zhen, L. Photodiode-Like Behavior and Excellent Photoresponse of Vertical Si/Monolayer $\mathrm{MoS}_{2}$ Heterostructures. Sci. Rep. 2014, 4, 7186.

53. Li, F.; Qi, J.; Xu, M.; Xiao, J.; Xu, Y.; Zhang, X.; Liu, S.; Zhang, Y. Layer Dependence and Light Tuning Surface Potential of 2D MoS 2 on Various Substrates. Small 2017, 13, 1603103.

54. Berweger, S.; Weber, J. C.; John, J.; Velazquez, J. M.; Pieterick, A.; Sanford, N. A.; Davydov, A. V.; Brunschwig, B.; Lewis, N. S.; Wallis, T. M., et al. Microwave near-Field Imaging of Two-Dimensional Semiconductors. Nano Lett. 2015, 15, 1122-1127.

55. Kelvin Probe Force Microscopy: Measuring and Compensating Electrostatic Forces; Sadewasser, S.; Glatzel, T. Eds.; Springer Science \& Business Media: Berlin, Germany, 2011. 56. Zhang, Z.; Yates, J. T., Jr. Band Bending in Semiconductors: Chemical and Physical Consequences at Surfaces and Interfaces. Chem. Rev. 2012, 112, 5520-5551.

57. Loppacher, C.; Zerweck, U.; Teich, S.; Beyreuther, E.; Otto, T.; Grafstrom, S.; Eng, L. M. FM Demodulated Kelvin Probe Force Microscopy for Surface Photovoltage Tracking. Nanotechnology 2005, 16, S1-S6.

58. Tonndorf, P.; Schmidt, R.; Böttger, P.; Zhang, X.; Börner, J.; Liebig, A.; Albrecht, M.; Kloc, C.; Gordan, O.; Zahn, D. R. T., et al. Photoluminescence Emission and Raman Response of Monolayer $\mathrm{MoS}_{2}, \mathrm{MoSe}_{2}$, and WSe 2 . Opt. Express 2013, 21, 4908-4916.

59. Lee, C.; Yan, H.; Brus, L. E.; Heinz, T. F.; Hone, J.; Ryu, S. Anomalous Lattice Vibrations of Single- and Few-Layer $\mathrm{MoS}_{2}$. ACS Nano 2010, 4, 2695-2700.

60. Li, H.; Zhang, Q.; Yap, C. C. R.; Tay, B. K.; Edwin, T. H. T.; Olivier, A.; Baillargeat, D. From Bulk to Monolayer $\mathrm{MoS}_{2}$ : Evolution of Raman Scattering. Adv. Funct. Mater. 2012, 22, 13851390.

61. Luo, X.; Zhao, Y. Y.; Zhang, J.; Toh, M. L.; Kloc, C.; Xiong, Q. H.; Quek, S. Y. Effects of

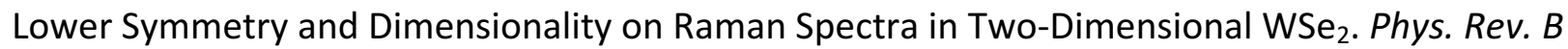
2013, 88, 195313.

62. Zhao, W.; Ghorannevis, Z.; Amara, K. K.; Pang, J. R.; Toh, M.; Zhang, X.; Kloc, C.; Tan, P. H.; Eda, G. Lattice Dynamics in Mono- and Few-Layer Sheets of $\mathrm{WS}_{2}$ and WSe 2 . Nanoscale 2013, 5, 9677-9683.

63. Chiu, K.-C.; Huang, K.-H.; Chen, C.-A.; Lai, Y.-Y.; Zhang, X.-Q.; Lin, E.-C.; Chuang, M.-H.; Wu, J.-M.; Lee, Y.-H. Synthesis of In-Plane Artificial Lattices of Monolayer Multijunctions. Adv. Mater. 2017, 1704796.

64. Chen, J.; Zhou, W.; Tang, W.; Tian, B.; Zhao, X.; Xu, H.; Liu, Y.; Geng, D.; Tan, S. J. R.; Fu, W., et al. Lateral Epitaxy of Atomically Sharp WSe $2 / \mathrm{WS}_{2}$ Heterojunctions on Silicon Dioxide Substrates. Chem. Mater. 2016, 28, 7194-7197. 


\section{TOC Graphic}

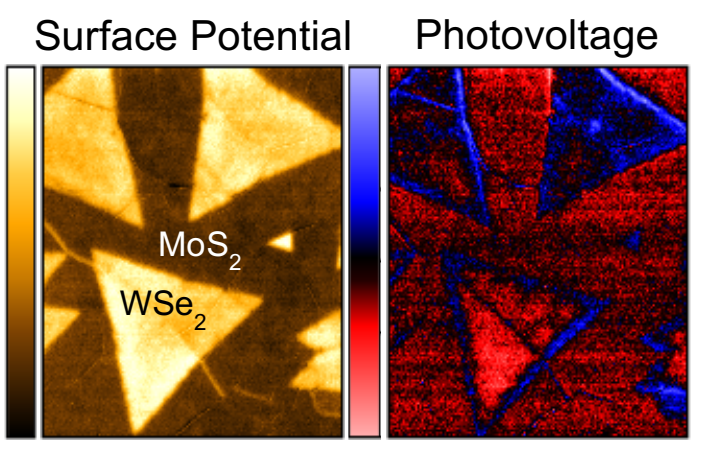

